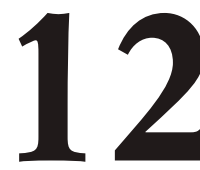

\title{
LA EDUCACIÓN EN VALORES EN LOS ESTUDIOS OFICIALES DE DANZA Y EN EL FOLKLORE ${ }^{1}$
}

\author{
(VALUES EDUCATION IN THE OFFICIAL DANCE STUDIES AND IN \\ FOLKLORE)
}

Rosario Rodríguez Lloréns

Instituto Superior de Enseñanzas Artísticas de la Comunitat Valenciana

\section{RESUMEN}

Con la intención de subsanar la incoherencia que presentan actualmente los estudios reglados de danza, en cuanto a las excelentes posibilidades educativas que ésta puede brindar al ser humano y la carencia en formación en valores que a menudo se observa en las aulas de los centros oficiales, se realizan dos estudios descriptivos en dos ámbitos diferentes de la enseñanza de la danza: el oficial y el tradicional. La información obtenida fundamenta el planteamiento y desarrollo de una intervención educativa, que tiene como objetivo la elaboración de la Propuesta Didáctica de Educación en Valores en la Danza (EVD), que se presenta finalmente como valiosa herramienta educativa, apta para la enseñanza de la danza en los estudios oficiales.

\section{ABSTRACT}

In an attempt to rectify the incoherence which currently shows the regulated dance education, in terms of the excellent educational opportunities that it can provide to the human being and the gap in education in values that is often observed in the classrooms of the learning official centers, two descriptive studies are developed in two different areas of dance education: official and traditional. The information obtained is the basis of the raising and development of an educational intervention that aims the elaboration of the "Didactic Proposal for Dance Education in Values $(D E V)^{\prime \prime}$, which is introduced as valuable educational tool, suitable for dance education in official studies. 


\section{INTRODUCCIÓN}

La investigación que se presenta surge en el marco donde confluyen dos grandes pasiones que me han acompañado a lo largo de mi vida: la danza y la enseñanza. Precisamente, el amor por la danza, el interés por convertir su enseñanza en una ocasión de enriquecimiento completo para los alumnos que asisten a las clases de esta disciplina artística y la preocupación producida por el hecho de comprobar que los alumnos de los estudios oficiales presentaban una tendencia a la rivalidad y el egocentrismo que se acentuaba con el paso de los cursos al llegar a los estudios profesionales, fueron los puntos principales que motivaron la realización de la investigación.

Hasta ahora, la danza ha sido estudiada principalmente desde el punto de vista histórico y también desde la perspectiva de la evolución de la técnica y la coreografía, en forma de tratados y manuales de danza. El hecho de abordar un estudio científico de la danza desde el punto de vista educativo, resulta muy oportuno dada la escasez de investigaciones acerca de esta disciplina en general, y de la enseñanza de la misma, en particular.

Por una serie de complejas circunstancias legislativas, los estudios de danza en el marco de los estudios superiores, no se implantaron en España hasta ya iniciado el siglo XXI en el que nos encontramos ${ }^{2}$, y este hecho ha dificultado el desarrollo de una labor de investigación que hubiera podido colaborar en el avance de los diferentes campos en los que la danza se ubica. La investigación realizada desea contribuir a la mejora del ámbito de la formación pedagógica del profesorado de danza, tanto de los actuales maestros como de aquellos alumnos que se están preparando para serlo en la especialidad de Pedagogía en el Grado en Pedagogía de la Danza.

La presente investigación, tuvo su antecedente en un trabajo previo titulado La competitividad en las clases del Grado Elemental de Danza ${ }^{3}$. Los resultados de esta primera investigación, que se limitó al Grado Elemental y al profesorado de los centros oficiales de la ciudad de Valencia, evidenciaron que se otorgaba la prioridad a la formación técnica y artística del alumnado frente a la educación en valores del mismo, demostrándose que en la medida en que el profesorado daba más importancia a la formación en valores de los alumnos, disminuía el fomento de la competitividad en las clases y viceversa. Tras estos resultados se decidió seguir investigando en esta dirección, ampliando el campo de estudio al Grado Medio ${ }^{4}$ de danza y la muestra al profesorado de las tres provincias de la Comunitat Valenciana. Igualmente, se consideró interesante analizar el contexto de la enseñanza y práctica de la danza en el folklore ${ }^{5}$, con la esperanza de encontrar en este ámbito aquellos valores que, inherentes a la danza, han acompañado a la humanidad desde sus comienzos. 
Si se comprobaba que los estudios oficiales de danza presentaban una carencia formativa en valores y, además, se podía hallar en la práctica y trasmisión de la danza tradicional del folklore la educación moral anhelada, entonces se estaría en disposición de aplicar todo lo estudiado en la mejora de la educación en valores del alumnado en la enseñanza reglada de la danza. Siguiendo a Pring, (2005, 158-159), estaríamos ayudando a nuestros alumnos a desarrollar, además de las habilidades de la danza, otras cualidades, como podrían ser las "cualidades morales y personales que podrían dirigir el uso de esas habilidades”.

\section{OBJETIVO Y PLANTEAMIENTO}

La finalidad última, planteada al abordar esta investigación, se orientaba a elaborar un plan de formación para que la enseñanza de la danza, en el marco de los estudios oficiales, contribuya a la educación en valores de los alumnos y realice así una tarea verdaderamente humanizadora.

Tal como plantea Bouché $(2003,100)$, la educación se entiende como una educación en valores ${ }^{6}$ orientada "en vía siempre de lo valioso" desde todas las dimensiones de la persona, debiéndose establecer previamente qué valores son los que conforman esa persona ideal y cuál es el significado del término valor. De entre las muchas definiciones adecuadas de este concepto, destacamos la propuesta por Marín Ibáñez (1976, 14-21): "El valor es la perfección o dignidad que tiene lo real o que debe tener y que reclama de nosotros el adecuado juicio y estimación".

Esta investigación parte de la premisa de que el aprendizaje y la práctica de la disciplina de la danza, integrada en la educación artística, pueden contribuir a enriquecer el proceso educativo del ser humano como persona. Este proceso, así entendido, se considera inherente al ser humano en dos sentidos, el primero porque sólo los hombres educamos a nuestros semejantes y, el segundo, "porque únicamente podemos desarrollar una existencia plenamente humana si hemos tenido la oportunidad de acceder a la educación" (García Amilburu, 2003, 209).

No obstante, con el objeto de fundamentar este supuesto, el desarrollo de la investigación se inició con la elaboración del marco de referencia teórico en el que se efectuó un recorrido por la Historia de la danza, primeramente en cuanto a su evolución y más adelante, en cuanto a la enseñanza de la misma tanto en el ámbito oficial como en el contexto del folklore, en España y en la Comunitat Valenciana ${ }^{7}$. Este estudio preliminar permitió comprobar dos circunstancias importantes para la investigación: $1^{\circ}$ Que se han atribuido a la danza funciones muy importantes en la educación y forma- 
ción moral del ser humano, pero también que el surgimiento de la danza profesional vino acompañado de la aparición de la competitividad entre los bailarines y que la danza corre el peligro de convertirse en un mero despliegue de proezas e inusitadas habilidades cuando se maximiza la importancia de la formación técnica del bailarín en detrimento de su formación artística y moral; $2^{\circ}$ Se observó que la enseñanza y práctica de la danza en el folklore seguiría cumpliendo en la actualidad una clara función social trasmitiendo una serie de importantes valores tales como el respeto, la solidaridad, la tolerancia o la unidad.

Sobre la primera de estas circunstancias, conviene resaltar que las enseñanzas artísticas, en sus diferentes ámbitos, han sido consideradas por numerosos autores como un medio óptimo para la educación de las personas, resaltándose diversos aspectos:

- Los beneficios educativos que el alumnado obtiene desarrollando su expresión a través de la educación por el arte (Read, 1986).

- El desarrollo que las enseñanzas de las artes producen en el juicio crítico de los alumnos abriendo sus mentes hacia otras realidades posibles (Eisner, 2002).

- La influencia que la educación artística tiene en el aumento de las fuentes de referencias de los niños que desarrollan así su capacidad creadora, su madurez y desarrollo general, convirtiéndose en individuos bien adaptados a su medio y capaces a su vez de mejorarlo creativamente en el futuro (Vygotskii, 1982 y Lowenfeld, 1961).

- La relevancia de la educación en el arte en la evolución de la percepción visual y el posterior desarrollo de valores emotivos, sensitivos y psicológicos (Arnheim, 1967).

Asimismo, el comunicado emitido en el XVII Encuentro de Consejos Escolares Autonómicos y del Estado, celebrado en Baleares en el año 2007, señala que las Enseñanzas Artísticas, entre las que se encuentra la danza, tienen una importancia extraordinaria "para la formación y el bienestar de las personas, por el conjunto de valores cognitivos, afectivos y de estímulo de las capacidades expresivas, creativas y emocionales que aportan" así como contribuyen a "los valores sociales, siendo instrumento de socialización, de comunicación, de inclusión, de participación”.

En cuanto a la segunda circunstancia constatada en el marco teórico, acerca del beneficio del estudio y la práctica de la danza del folklore, destacamos que el valor pedagógico del patrimonio fue reivindicado por el Con- 
sejo de Europa en su Recommendation $N^{o} R$ (98) 5, relativa a la pedagogía del patrimonio, apreciada como "medio privilegiado de dar sentido al futuro mediante una mejor comprensión del pasado". Ya en 1995, en el Seminar de Brussels del Consejo de Europa, se animaba a desarrollar en los jóvenes, a través de actividades de educación del patrimonio, el interés por luchar "contra todas las formas de intolerancia y de participar activamente en la construcción de una sociedad europea basada en valores comunes, caracterizada por la democracia, la tolerancia y la solidaridad”.

La enseñanza de la danza y bailes tradicionales forma parte actualmente de los planteamientos curriculares de la Comunitat Valenciana en la Educación Infantil, en las áreas de los Lenguajes: comunicación y representación; en la Educación Primaria, en las áreas de conocimiento de la Educación Artística y la Educación Física; y en la Educación Secundaria Obligatoria, en las materias de la Educación Física y la Música. Estas propuestas concuerdan con la opinión de Pujol y Serra $(1998,13)$, para el que la enseñanza de la danza tradicional, está recomendada en el currículo de Música, por "la adecuación del movimiento corporal al estímulo sonoro" y en la Educación Física, porque la danza es "un lenguaje que se percibe y expresa a través del cuerpo a partir de su propio movimiento y de su respiración, en el tiempo y en el espacio".

Tras la elaboración del Marco teórico, este trabajo se estructuró en tres partes: la Fase Descriptiva, la Fase de Evaluación e Intervención Didáctica y la presentación de la Propuesta Didáctica de Educación en Valores en la danza $(E V D)$.

\section{METODOLOGÍA Y DESARROLLO DE LA FASE DESCRIPTIVA}

En el desarrollo de la Fase Descriptiva se analizó, por una parte, la enseñanza de la danza en los estudios oficiales en un marco amplio buscando establecer con mayor certeza si los resultados obtenidos en el trabajo citado La Competitividad en las clases del Grado Elemental de Danza, son representativos de este contexto educativo y, por otra, la enseñanza de la danza en el folklore intentando descubrir cuáles son las características educativas beneficiosas que están presentes en ella que puedan ayudar a lograr el objetivo último del estudio.

\subsection{Diseño de investigación en el ámbito de la enseñanza oficial de la danza}

Este primer diseño, se enmarcaba en el paradigma empírico-analítico, escogiéndose como método de investigación la realización de un 
estudio descriptivo sobre el profesorado de danza en referencia a la trasmisión de valores en sus aulas y el fomento de la competitividad en las mismas.

Se establecieron como variables del estudio las siguientes: Formación Técnica, Formación Artística y Formación en Valores, todas ellas consideradas valores a trasmitir y Fomento de la Competitividad, entendida como un contravalor.

El planteamiento del estudio descriptivo de los estudios oficiales de danza continúa con la formulación de las hipótesis del estudio, que apuntan a la existencia de un desequilibrio en cuanto a la importancia que el profesorado de los estudios oficiales de danza concede a la formación técnica y artística del alumnado en detrimento de la trasmisión de valores morales, por una parte, y hacia el descenso de la importancia que este mismo profesorado confiere en el paso del Grado Elemental al Grado Medio, a la variable Formación en Valores frente al aumento de las variables Formación Técnica, Formación Artística y Fomento de la Competitividad, por otra.

Para la obtención de la información pertinente que posibilitara la comprobación de estas hipótesis se construyó un instrumento original de medida el -Cuestionario de Valores y Competitividad en la Danza (CVCD)-, aportación muy provechosa tanto para este estudio como para posibles investigaciones futuras, dada la carencia actual de herramientas de evaluación para la danza.

El Cuestionario-CVCD, que fue aplicado al profesorado de los centros de estudios oficiales de danza de las tres provincias de la Comunitat Valenciana, contiene los ítems correspondientes a las cuatro variables citadas, presentados de forma aleatoria. Los ítems suponen la definición operativa de cada uno de los indicadores representativos del sentido de las variables a las que pertenecen y están redactados siguiendo las directrices de la construcción de instrumentos tipo Escalograma de Guttman (García Ferrando, 1986). En este tipo de cuestionarios, los ítems se redactan de manera que expresen de forma creciente una actitud favorable hacia la variable representada en el continuo, de forma que, si una persona responde que está de acuerdo con un ítem debería estar de acuerdo con todos los ítems que están por debajo de ese ítem en el continuo.

Con la intención de profundizar en la obtención de escalas de posicionamiento del profesorado de danza, en cuanto a la importancia que éstos dan a los diferentes valores y contravalores que se fomentan en las clases, por un lado, y de comprobar que el cuestionario elaborado cumplía los 
requisitos de validez y fiabilidad requeridos para la investigación, por otro, las respuestas del profesorado de la muestra se analizaron con el programa MINISTEP según el modelo de Rasch, para las cuatro variables del estudio.

La técnica de Rasch aproxima los puntajes crudos a puntajes lineales obteniendo una escala lineal de datos estándar, aptos para la aplicación de las operaciones matemáticas significativas para el estudio.

El Cuestionario-CVCD superó las diferentes pruebas de fiabilidad y validez, cumpliendo las dos características principales del modelo: la suficiencia de la puntuación total, que sostiene que en la respuesta de una persona a un ítem solamente influyen dos parámetros: la dificultad del ítem y la habilidad del sujeto respondente (Wright y Stone, 1998) y la condición de la unidimensionalidad, pues se asume que los ítems de la prueba responden ante indicadores de la misma variable (Oreja Rodríguez, 2005).

El análisis realizado posibilitó la elaboración de unas Tablas de Conversión que permitieron asignar a cada una de las puntuaciones iniciales de las respuestas de los profesores una puntuación final lineal para cada una de las variables, con lo cual el Cuestionario- $C V C D$ quedó configurado como un instrumento de fácil utilización e interpretación. Las medias de estas puntuaciones finales se muestran en la siguiente Tabla 1:

\begin{tabular}{|c|c|c|c|c|}
\hline & \multicolumn{2}{|c|}{ GRADO ELEMENTAL } & \multicolumn{2}{c|}{ GRADO MEDIO } \\
\hline VARIABLES & MEDIA & DT & MEDIA & DT \\
\hline Formación Técnica & 78,4 & 18 & 90,9 & 13,1 \\
\hline Formación Artística & 72,7 & 16,3 & 87,4 & 13,4 \\
\hline Formación en Valores & 73,5 & 15,5 & 68,2 & 11,5 \\
\hline Fomento de la Competitividad & 33,7 & 13,2 & 40,9 & 9,7 \\
\hline
\end{tabular}

Tabla 1. Puntuaciones medias finales y desviaciones típicas de los profesores en los Grados Elemental y Medio

Con estos resultados se hizo una comparativa de la importancia que los profesores atribuyen a la trasmisión de los diferentes valores y contravalores tanto en el Grado Elemental como en el Grado Medio de danza, que podemos observar en los siguientes Gráficos 1 y 2: 


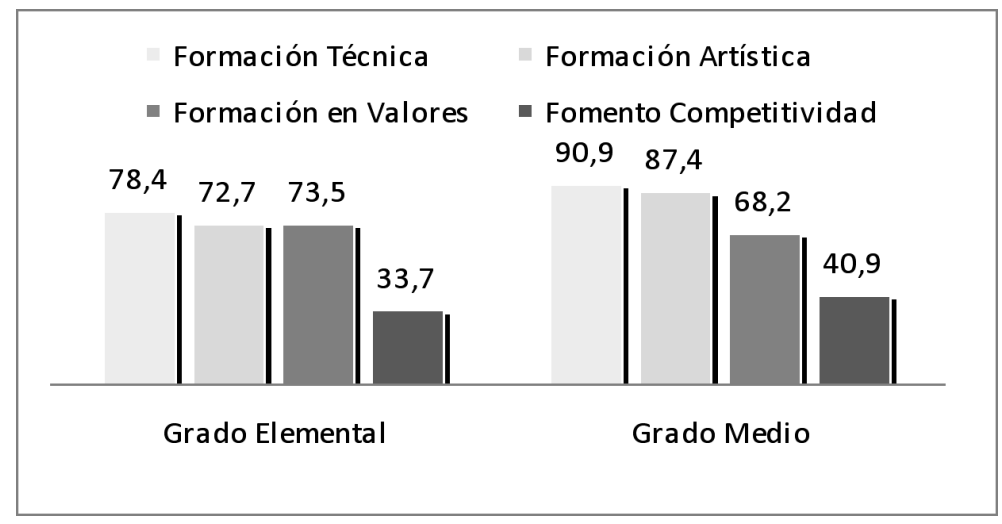

Gráfico 1. Valores medios de las variables en los Grados Elemental y Medio

Vemos claramente en el Gráfico1, cómo los valores alcanzados por las cuatro variables en el Grado Medio respecto del Grado Elemental, suponen un aumento de las puntuaciones de las variables Formación Técnica, y Formación Artística. Sin embargo, la variable Formación en Valores sufre un descenso, y la variable Fomento de la Competitividad experimenta un aumento.

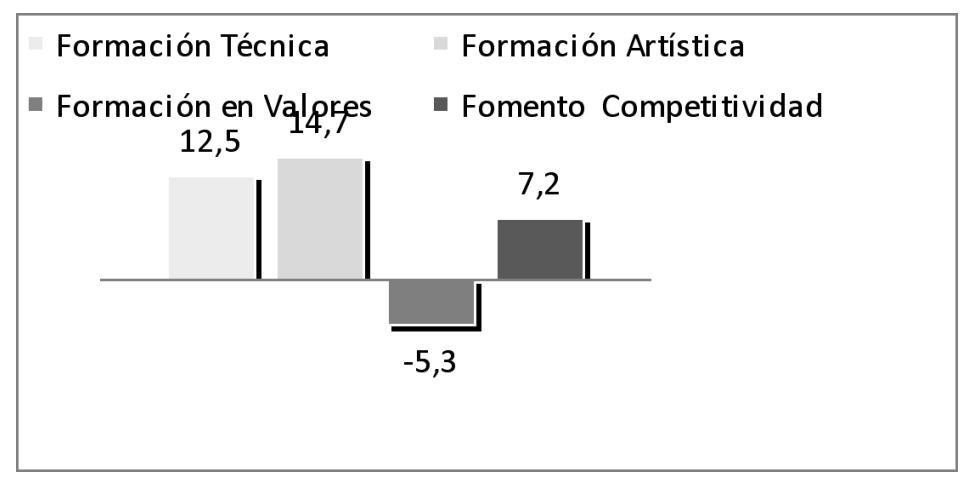

Gráfico 2. Variación de los valores medios de las variables en los Grados Elemental y Medio

El Gráfico 2 permite apreciar mejor la variación sufrida, en cuanto a la importancia que los profesores conceden a las cuatro variables del estudio en el paso del Grado Elemental al Grado Medio. La variable Formación Artística es la que experimenta la mayor variación al alza con un aumento de 14,7 puntos, seguida de la variable Formación Técnica que aumenta 12,5 puntos y de la variable Fomento de la Competitividad que sube 7,2 puntos. Sin embargo, la variable Formación en Valores sufre una variación a la baja con un descenso en su valor medio de 5,3 puntos. 
Sobre estos datos se realizó el Test de Kruskal-Wallis pudiéndose afirmar que los conjuntos de datos de las variables Formación Técnica (Test statistic $=18,5$, P-Value $=0,000017)$; Formación Artística $($ Test statistic $=27,66$, PValue $=1,44 \mathrm{E}-7$ ); y Fomento de la Competitividad (Test statistic $=9,87$, $\mathrm{P}$-Value $=0,00168)$, son diferentes en el Grado Elemental y en el Grado Medio, con un 99\% de confianza, al igual que en el caso de la variable Formación en Valores (Test statistic $=3,6073, \mathrm{P}$-Value $=0,05752)$, con un 90\% de confianza. Estos resultados son muy preocupantes pues confirman empíricamente, con significación estadística, la tendencia que presenta el ámbito profesional de la danza a la aparición de la competitividad, que ya pudimos apreciar en la revisión de la evolución de la danza y sus funciones a lo largo de la historia.

Asimismo, es de destacar que en el análisis se observó que en ambos grados el valor de la cooperación obtenía las puntuaciones más bajas por parte del profesorado, siendo muy acusado el descenso de la importancia que se atribuye a este valor en el Grado Medio.

Tras estas conclusiones, se estuvo en condiciones de confirmar las hipótesis planteadas en el diseño de este apartado, habiendo quedado demostrado que, en relación con el Grado Elemental, la importancia concedida por el profesorado de danza a la formación en valores del alumnado, disminuye en el Grado Medio al mismo tiempo que se incrementa el fomento de la competitividad en las clases y se valora más la formación técnica y artística. Por otra parte, se debe especificar que en el Grado Elemental, no existe el desequilibrio entre la formación técnica y artística de los alumnos frente a la formación en valores morales de los mismos, que sí aparece en el Grado Medio.

\subsection{Diseño de investigación en el ámbito de la enseñanza y práctica de la danza en el folklore}

El segundo diseño realizado en esta fase descriptiva, se encuadraba en el paradigma interpretativo de investigación realizándose una microetnografía educativa (Goetz y Lecompte, 1988), para averiguar y comprender lo mejor posible aquello que acontece en la práctica y enseñanza del folklore desde el punto de vista de sus principales protagonistas, los profesores y bailadores de los grupos de danzas, en lo referente a los valores y a su trasmisión.

La estrategia cualitativa que se utilizó para obtener la información deseada fue la observación participante del Grup de Danses Alimara de la Sociedad Coral El Micalet de Valencia. El periodo de observación participante 
se prolongó durante un año, en el que se asistió en total a 32 sesiones de ensayos del grupo, además de acompañar al mismo en más de 10 actividades distintas. La participación de la autora en las diferentes actividades como bailadora-investigadora fue activa desde el primer momento, como un componente más del grupo, dada la extraordinaria acogida ofrecida por todos sus miembros.

Para el análisis cualitativo de la información que se iba obteniendo, se utilizó el programa ATLAS.ti ${ }^{8}$ que parte de las premisas del método de la Teoría Fundamentada (Sandín, 2003). Dentro de las posibilidades que el programa ATLAS.ti ofrece, se escogieron los diagramas o networks como soporte visual y práctico para exponer los descubrimientos obtenidos en relación con el ámbito de la enseñanza y práctica de la danza en el folklore, en forma de citas, códigos, familias de códigos, categorías centrales, etc. Se elaboraron para ello diversas networks sobre las familias de códigos: Objetivos de los grupos de folklore y El profesorado de folklore infantil y juvenil, y en torno a las categorías o temas centrales: Valores en el folklore y Valores en la enseñanza infantil y juvenil del folklore (Cfr. Rodríguez Lloréns, 2009, 206222), de las cuales mostramos en la Figura 1, a modo de ejemplo, la network representativa de la familia central: Objetivos en los grupos de folklore en la que se muestran los tres objetivos principales que orientan la labor de los grupos, expresados en los códigos: investigación, difusión y recuperación de tradiciones. A su vez, cada uno de estos códigos se vincula con otros, quedando apuntado en la network el carácter de la relación que los une.

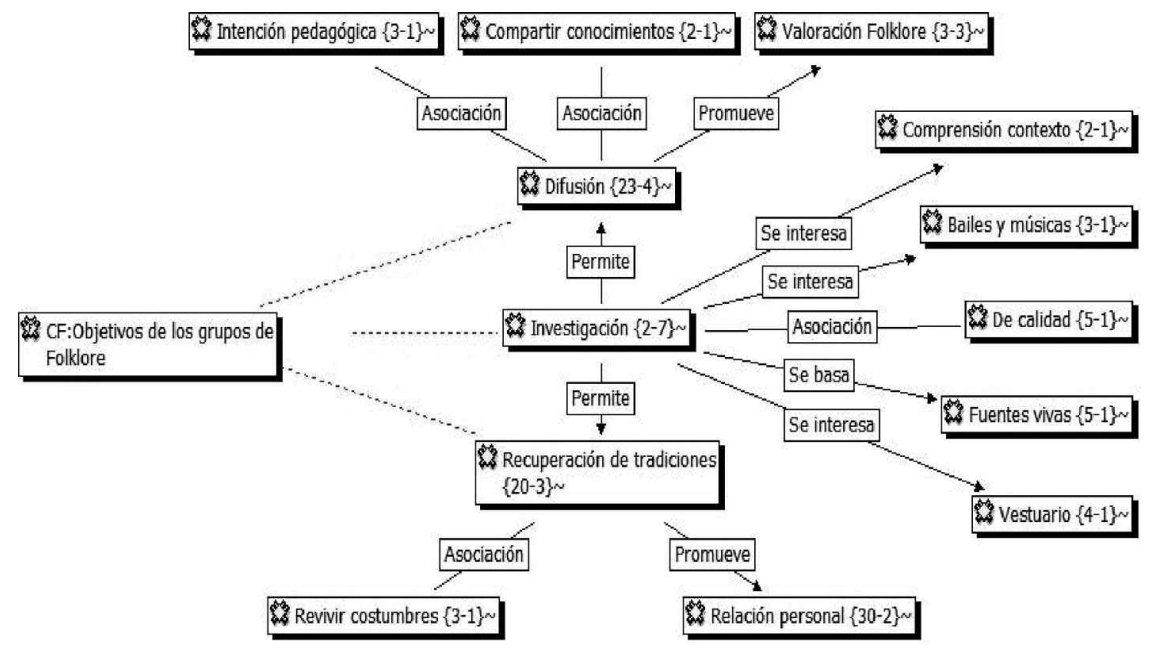

Figura 1. Network: Objetivos de los grupos de folklore 
Para una mejor comprensión de los símbolos del diagrama, podemos ver en la siguiente Tabla 2, el significado de los textos y cifras que aparecen en el mismo:

\begin{tabular}{|c|l|l|}
\hline \multicolumn{3}{|c|}{ CÓDIGO } \\
\hline Texto & Nombre del código \\
\hline $\mathrm{n} 1$ & Número de citas relacionadas con el código \\
\hline $\mathrm{n} 2$ & Número de vínculos del código \\
\hline$\sim$ & El código tiene un comentario \\
\hline \multicolumn{3}{|l|}{ FAMILIA DE CÓDIGOS } \\
\hline Texto & \multicolumn{2}{|l|}{ Nombre de la familia de códigos } \\
\hline
\end{tabular}

Tabla 2. Significado de las leyendas de los símbolos en las networks (Muñoz, 2005, 37).

Tras el proceso de teorización del análisis se llevo a cabo un proceso de triangulación de los datos obtenidos, tras el cual se obtuvieron las siguientes conclusiones: en el ámbito de la enseñanza y práctica de la danza en el folklore sí se lleva a cabo una educación en valores, y se contribuye a la formación integral de la persona, puesto que en este contexto:

- Se despierta la consciencia de herencia y arraigo cultural.

— Se fomentan principalmente los valores de la unidad y la cooperación.

- Se potencia la expresión de la alegría y la felicidad, disfrute y satisfacción en la actividad compartida.

- Está prácticamente ausente el fomento de la competitividad que sólo aparece en la faceta más profesional del folklore.

- La motivación de los jóvenes bailadores se basa en la convivencia con los amigos y el disfrute de bailar.

\section{METODOLOGÍA Y DESARROLLO DE LA FASE DE EVALUACIÓN E INTERVENCIÓN DIDÁCTICA}

Las conclusiones halladas en la fase descriptiva, sirvieron de base para abordar una nueva fase de la investigación, la fase de evaluación e inter- 
vención didáctica en la que se diseñó un Proyecto de Propuesta Didáctica de Educación en Valores en la Danza en la que se buscaba el fomento de los valores que se trasmiten en el ámbito del folklore y se emulaba la forma en que los profesores de los niños y jóvenes tienen de trasmitirlos. Además, como el aspecto menos valorado en el estudio descriptivo de la enseñanza oficial de la danza fue la "cooperación", se decidió utilizar la metodología del Aprendizaje Cooperativo en la elaboración de diversas actividades y juegos educativos.

El objetivo general de esta fase consistía en realizar una intervención educativa con un grupo de alumnos de estudios oficiales de danza, que permitiera la elaboración de una Propuesta Didáctica de Educación en Valores en la Danza, que potenciase la formación en valores y contrarrestara la competitividad entre los alumnos.

La experiencia se desarrolló, a modo de Diseño cuasiexperimental de pretest-posttest con grupo de control no equivalente (Bisquerra, 2004), a lo largo de un curso lectivo completo en la asignatura Danza Española y contó con la colaboración de dos grupos de alumnas de $4^{\circ}$ curso de Grado Elemental, curso preparatorio de los estudios profesionales, de dos escuelas diferentes: las alumnas del Centre Elemental de Dansa Botànic, Espai de Dansa, de Valencia, como grupo experimental y las alumnas del Conservatorio Profesional de Danza de Valencia, como grupo control. El grupo experimental asistió a las clases en las que se ensayaba el Proyecto de la Propuesta Didáctica, mientras que el grupo control asistió a las clases de danza regulares en su centro de estudios.

Se establecieron como variables del diseño las siguientes: Autoestima, Cooperación, Responsabilidad y Tolerancia, que se correspondían con aquellos valores que se deseaba potenciar con la Propuesta Didáctica-EVD.

Las hipótesis propuestas en el estudio experimental sostienen que la enseñanza oficial de la danza es posible educando en valores al alumnado sin estimular la competitividad, de modo que, tras la experiencia educativa de la Fase de Evaluación e Intervención Didáctica, las alumnas del grupo experimental habrán evolucionado mejor que el alumnado del grupo control en cuanto a los valores planteados, además de haberse formado satisfactoriamente en su faceta de bailarinas.

La aplicación del Proyecto de Propuesta Didáctica al grupo experimental pudo ser llevada a cabo personalmente por la autora de la investigación, dado su desempeño docente habitual. La experiencia educativa se desarrolló de forma sumamente satisfactoria. Las lógicas preocupaciones acerca de la efectividad que esta novedosa propuesta metodológica podía te- 
ner en la enseñanza de la danza, a nivel de los exigentes contenidos curriculares de los estudios reglados, fueron desapareciendo gradualmente al constatar que las alumnas progresaban de forma evidente en sus habilidades dancísticas al mismo tiempo que mostraban en las clases una alta motivación. Es de destacar asimismo, la felicidad que se observaba en las alumnas que bailaban con una expresión alegre y espontánea similar a la que se había observado en los niños y jóvenes que participaban en las actividades de danza tradicional.

Junto con la evaluación curricular propia de los estudios reglados de danza, se efectuaba de forma continua la valoración cualitativa del desarrollo del Proyecto que permitía la mejora constante del mismo. Al finalizar el curso y, con él, la intervención educativa, todas las alumnas del grupo experimental aprobaron el curso de danza y aquellas que deseaban continuar sus estudios profesionales en la especialidad de Danza Española, se presentaron a la Prueba de Acceso para Grado Medio en dos centros oficiales en los que se imparte este nivel educativo. La prueba fue superada por el $100 \%$ de las alumnas presentadas, que obtuvieron plaza en los centros deseados.

En cuanto a la evaluación cuantitativa del diseño experimental, para obtener la información necesaria sobre las variables del estudio: Autoestima, Cooperación, Responsabilidad y Tolerancia, se recurrió a diversos instrumentos, elaborados o adaptados al efecto, que se aplicaron sobre los grupos experimental y control en dos ocasiones -Pretest y Posttest- al principio y al final del curso, respectivamente. En la siguiente Tabla 3, se ofrecen las puntuaciones medias obtenidas por ambos grupos en las dos valoraciones:

\begin{tabular}{|c|c|c|c|c|c|c|c|c|}
\hline & \multicolumn{2}{|c|}{ GRUPO } & \multicolumn{2}{c|}{$\begin{array}{c}\text { GRUPO } \\
\text { CONTROL }\end{array}$} & \multicolumn{2}{c|}{$\begin{array}{c}\text { GRUPO } \\
\text { EXPERIMENTAL }\end{array}$} & \multicolumn{2}{c|}{$\begin{array}{c}\text { GRUPO } \\
\text { CONTROL }\end{array}$} \\
\hline VARIABLES & MEDIA & DT & MEDIA & DT & MEDIA & DT & MEDIA & DT \\
\hline Autoestima & 20,33 & 2,74 & 21,44 & 2,60 & 19,56 & 2,65 & 22,0 & 1,94 \\
\hline Cooperación & 25,44 & 2,70 & 27,67 & 2,40 & 25,67 & 1,41 & 24,89 & 1,76 \\
\hline Responsabilidad & 24,33 & 3,04 & 27,78 & 2,49 & 23,67 & 2,5 & 26,0 & 2,92 \\
\hline Tolerancia & 26,22 & 2,05 & 27,89 & 1,54 & 24,89 & 2,57 & 26,56 & 2,35 \\
\hline & \multicolumn{6}{c|c|}{ PRETEST } & \multicolumn{5}{c|}{ POSTTEST } \\
\hline
\end{tabular}

Tabla 3. Puntuaciones medias y desviaciones típicas de las variables Autoestima, Cooperación, Responsabilidad y Tolerancia, en las valoraciones Pretest y Posttest 
La variación en la puntuación de las variables obtenida en la valoración Posttest, respecto de la alcanzada en la valoración Pretest, fue desigual para ambos grupos, experimental y control. La diferencia entre ambas variaciones se puede apreciar en el Gráfico 3 que mostramos a continuación:

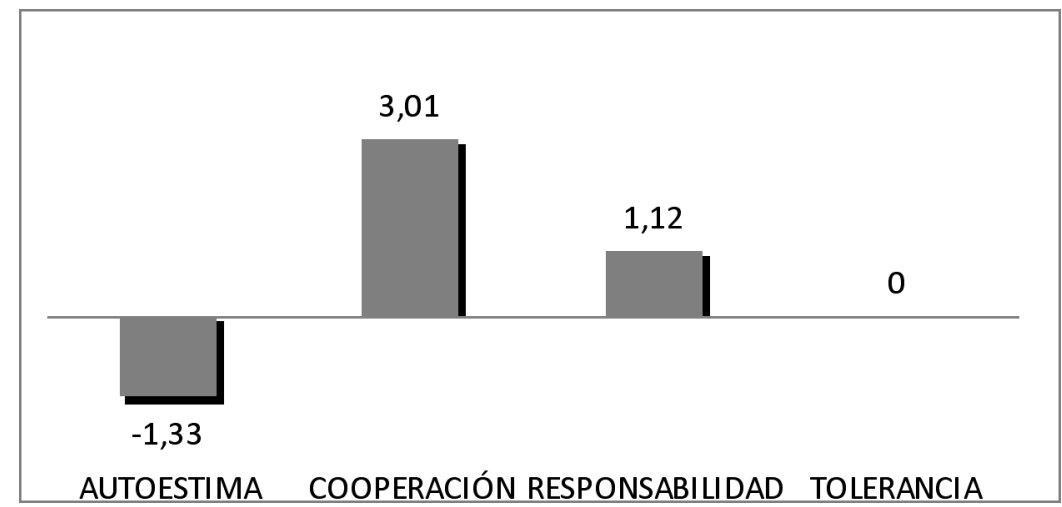

Gráfico 3. Diferencia en la variación de la puntuación de las variables entre el grupo experimental y el grupo control

La diferencia en la variación de las puntuaciones para la variable Autoestima entre ambos grupos ha sido negativa de $-1,33$ puntos. Este resultado se considera correcto en este estudio puesto que en la primera evaluación Pretest, ambos grupos se situaban en la franja considerada de autoestima elevada. Sin embargo, tras la intervención educativa, el grupo experimental se localizó en la franja de puntuación correspondiente a una autoestima equilibrada, mientras que el grupo control tuvo un ascenso no aconsejable en la franja de la autoestima elevada que, según Baumeister $(1999,241)$ conlleva también connotaciones negativas tales como "orgullo, egotismo, arrogancia, narcisismo, y sentido de la superioridad". Estos problemas no son ajenos a la danza pues en ésta aparece frecuentemente la orientación al ego referida en la Teoría de la perspectiva de la meta o de los logros (Nicholls, 1984, 1989), que cuando se manifiesta de forma excesiva "es fácil que se piense que hay que ganar a toda costa, superar a los demás bailarines, incluso usando malas artes, técnicas engañosas, etc." (Fuentes, 2007, 34).

En cuanto a la variable Cooperación, observamos una diferencia positiva de 3,01 puntos a favor del grupo experimental en relación con el grupo control, siendo significativa estadísticamente con un 95\% de confianza (ANOVA: F- Ratio: 4,94, P-Value: 0,0410). Por lo que respecta a la variable Responsabilidad, vemos que la diferencia es de nuevo positiva para el grupo 
experimental, en este caso de 1,12 puntos. En el caso de la variable Tolerancia, la variación en la puntuación de ambos grupos ha sido idéntica.

Los resultados obtenidos en la Fase de Evaluación e Intervención Didáctica, pese a no ser contundentes, sí son esperanzadores en cuanto al beneficioso impacto formativo que puede surtir de la aplicación de la Propuesta Didáctica-EVD en su versión definitiva, pues ésta se administró sólo a modo de ensayo al encontrarse aún por perfilar. Se confirman así las hipótesis planteadas en esta Fase, puesto que el alumnado del grupo experimental, tras la intervención educativa, obtuvo mejores resultados en las variables-valores estudiados que el alumnado del grupo control, a excepción de la variable Tolerancia que no experimentó una variación diferente en ambos grupos. Afirmamos, por tanto, que es posible la enseñanza oficial de la danza en el Grado Elemental educando en valores, sin estimular la competitividad.

\section{PRESENTACIÓN DE LA PROPUESTA DIDÁCTICA DE EDUCACIÓN EN VALORES EN LA DANZA (EVD)}

Como resultado directo del proceso de intervención educativa realizado en la fase anterior, se presentó la Propuesta Didáctica de Educación en Valores en la danza (EVD). La Propuesta-EVD incluía una Introducción y los apartados correspondientes a: Justificación, Objetivos actitudinales, Metodología y Evaluación.

Los objetivos pretenden el desarrollo de la formación de los alumnos del Grado Elemental de danza en los valores: autoestima, cooperación, responsabilidad y tolerancia, apostando por una educación moral del alumnado con el fin de que mejore como persona al mismo tiempo que se instruye en la disciplina de la danza.

La metodología empleada en la Propuesta Didáctica de Educación en Valores en la Danza (EVD) está basada, como ya dijimos, en la actuación observada en el profesorado de la danza en el folklore y en las directrices del Aprendizaje Cooperativo.

La Propuesta Didáctica-EVD se presenta acompañada de una colección de 16 fichas de actividades cooperativas de enseñanza-aprendizaje de la danza (Cfr. Rodríguez Lloréns, 2009, pp. 303-318), diseñadas específicamente para la Propuesta Didáctica-EVD ${ }^{9}$. Entre las mismas, se encuentran actividades de Rompecabezas de Aronson, dados los beneficios educativos que comparten con otras técnicas cooperativas en cuanto a la autoestima, la creatividad, etc. (Díaz-Aguado, 2003); Actividades cooperativas con y sin objetivo cuantificable como pueden ser las de imitación y las de Reproducción 
de secuencias rítmicas (Velázquez, 2004, 46-47); y otras actividades ideadas para favorecer la creatividad: 1. De creación; 2. De comunicación; y 3. De simbolismo.

\section{CONCLUSIÓN FINAL}

Los resultados que se han obtenido durante la elaboración de la investigación, han afianzado el convencimiento acerca de la necesaria reflexión que los profesores de los estudios oficiales de danza, entre los que se incluye la autora de esta investigación, deben realizar sobre su responsabilidad en la formación moral de sus alumnos.

Las conclusiones a las que se ha ido llegando en las diferentes Fases del estudio realizado, confirman el gran interés que tienen los profesores de las enseñanzas regladas de danza, por enseñar lo mejor posible a los niños y jóvenes que acuden diariamente a sus clases. No obstante, estas conclusiones también evidencian que, al abordar el nivel profesional, son más propensos a estimular actitudes competitivas en las aulas y dan menos importancia a la formación en valores morales de los alumnos, aspectos que no se observan en el contexto de la enseñanza y práctica de la danza tradicional.

Lamentablemente, en el mundo profesional de la danza se vive en demasiadas ocasiones un ambiente competitivo que adolece de excesiva rivalidad y de falta de unión. Pero los docentes pueden ayudar a que esta situación cambie y recordar que la labor que desarrollan en sus centros, además de servir para formar brillantes profesionales, también debe colaborar en la educación de los futuros bailarines, coreógrafos, profesores, etc. como seres humanos completos. Y confiamos en que un modo de lograrlo puede ser la utilización de la herramienta aportada en esta investigación, que hemos llamado Propuesta Didáctica de Educación en Valores en la Danza-EVD, con la que se desea contribuir a que la danza siga siendo aquello que ella misma es por su propia naturaleza y lo que nunca debería dejar de ser: un medio maravilloso de creación, comunicación, perfección, belleza y crecimiento espiritual. 


\section{NOTAS}

1. Este artículo recoge algunos datos de la Tesis Doctoral titulada "La educación en valores a través de la danza en las enseñanzas regladas y en el folklore. Propuesta educativa para el ámbito de los estudios oficiales de danza". La Tesis fue dirigida por la Dra. D ${ }^{a}$ María García Amilburu, a la que expreso desde aquí mi gratitud.

2. En el año 1990, la LOGSE fue la primera disposición legal que reguló de forma unificada los estudios de danza en España, este significativo avance en la historia de las enseñanzas de danza en nuestro país, elevaba a la categoría de Licenciatura la titulación del Grado Superior de danza. Este Grado se implantó en la Comunitat Valenciana a través del Decreto 128/2002, de 30 de julio, del Gobierno Valenciano, por el que se establece el currículo de las enseñanzas del grado superior de Danza en la Comunitat Valenciana y se regula la prueba de acceso a estos estudios (DOGV N $\mathrm{N}^{\mathrm{H}}$ 4308, de 6 de agosto de 2002), cursándose actualmente en los Conservatorios Superiores de Danza, pertenecientes al ISEACV.

3. Trabajo del periodo de investigación, dentro del Programa de Doctorado Teoría de la Educación y Pedagogía Social, de la UNED, realizado bajo la dirección de la Dra. $\mathrm{D}^{\mathrm{a}} \mathrm{M}^{\mathrm{a}}$ Teresa Martín González.

4. Con fecha posterior a la finalización de la investigación, como consecuencia del desarrollo de los nuevos currículos de danza en el marco de la LOE, los grados de los estudios oficiales de danza -Grado Elemental y Grado Medio- pasaron a denominarse Enseñanzas Elementales y Enseñanzas Profesionales, respectivamente.

5. El término folk-lore fue acuñado por primera vez por William John Thoms (bajo el seudónimo de Ambros Merton) en 1840, con el significado de: "conocimiento del pueblo" (American Folklore Society, 1888). En 1971, Ben-Amos, formuló una nueva definición, ampliamente aceptada actualmente: "la comunicación artística en grupos pequeños".

6. Para una lectura más detallada acerca de de los valores y su historia en la educación en España, se recomienda el completo estudio realizado por Escámez, 2003.

7. Remitimos a la lectura de Rodríguez Lloréns, 2009, 31-105, para una mejor comprensión de este recorrido histórico, así como la consulta de la extensa bibliografía existente en relación con la Historia de la Danza, en general, de la cual destacaríamos la aportación de los siguientes autores: Abad, 2004; Bourcier, 1981; Christout, 1995; Ginot y Michel, 2002; Salazar, 2003; Steeh, 1982; y de la danza en el folklore español y valenciano, en particular: Capmany, 1946; Crivillé, 1983; Hoyos y Hoyos, 1985; Oller y Martí, 1998; Preciado, 1969.

8. Agrademos al profesor Juan Muñoz Justicia, de la Universitat Autònoma de Barcelona, la elaboración y puesta a disposición del público en general del excelente Manual del programa ATLAS.ti: Análisis cualitativo de datos textuales con ATLAS.ti5.

9. El desarrollo de algunas de estas actividades cooperativas se ha realizado adaptando a la danza juegos y experiencias de autores que han desarrollado estudios en variados contextos tales como: artes plásticas, dramatización, deportes, etc.: Garaigordobil, 2004; Omeñaca y Ruíz Omeñaca, 1999; Orlick, 1997. 


\section{REFERENCIAS BIBLIOGRÁFICAS}

Abad Carles, A. (2004). Historia del ballet y de la danza moderna. Madrid: Alianza Editorial.

American Folklore Society (1888). The Credit of Originating the Term FolkLore. The Journal of American Folklore, 1 (1), 79-81. Disponible en:

http://www.jstor.org/pss/532893 [consulta 2008, 17 de marzo]

Arnheim, R. (1967) Arte y percepción visual: psicológica de la visión creadora. Buenos Aires: Eudeba, Editorial Universitaria de Buenos Aires.

Baumeister, R.F. (ed.) (1999). The Self in Social Psychology. Philadelphia, PA: Psychology Press (Taylor \& Francis).

Ben-Amos, D. (1971). Toward a Definition of Folklore in Context. The Journal of American Folklore, 84 (33), 3-15. Disponible en: http://www.jstor.org/pss /539729 [consulta 2008, 16 de marzo]

Bisquerra Alzina, R. (coord.) (2004). Metodología de la investigación educativa. Madrid: La Muralla S.A.

Bouché Peris, J. H. (2003). Educar para un nuevo espacio humano. Madrid: Dikynson.

Bourcier, P. (1981). Historia de la danza en Occidente. Barcelona: Blume.

Capmany, A. (1946). El baile y la danza, en Carreras y Candi, F. (dir.) Folklore y costumbres de España. Barcelona: Casa Editorial Alberto Martín, Tomo II.

Christout, M - F. (1995). Le Ballet occidental. París: Desjonquères.

Consejos Escolares Autonómicos y del Estado (2007). Conclusiones del XVII Encuentro de Consejos Escolares Autonómicos y del Estado: Las Enseñan- zas Artísticas. Palma de Mallorca, 21 de abril de 2007.

Crivillé i Bargalló, J. (1983). El folklore musical, en Crivillé i Bargalló, J. Historia de la música Española. Madrid: Alianza Música, Tomo VII.

Díaz-Aguado, M. J. (2003). Educación intercultural y aprendizaje cooperativo. Madrid: Pirámide.

Eisner, E. (2002). The Arts and the Creation of Mind. New Haven: Yale University Press.

Escámez, J. (2003). Los valores y la educación en España: 1975-2001, en Ortega, P. (ed.) Teoría de la educación, ayer y hoy. Murcia: Seminario Interuniversitario de Teoría de la Educación, 205237.

Fuentes, S. (2007). Cuadernos de Danza $N^{o}$ 2: Motivación para bailarines. Bilbao: Asociación Cultural Danza Getxo.

Garaigordobil Landazabal, M. (2004). Juegos cooperativos y creativos para grupos de niños de 10 a 12 años. Madrid: Pirámide.

García Amilburu, M. (2003). El ser humano protagonista de la educación, en García Amilburu, M. (ed.) Claves de la filosofía de la educación. Madrid: Dykinson, 209-221.

García Ferrando, M. (comp.) (1986). El análisis de la realidad social. Métodos y técnicas de investigación. Madrid: Alianza Editorial.

Ginot, I. y Michel, M. (2002). La danse au XXe siècle. París: Larousse.

Goetz, J.P. y Lecompte, M.D. (1988). Etnografía y diseño cualitativo de investigación educativa. Madrid: Morata. 
Hoyos Sainz, L. y Hoyos Sancho, N. (1985). Manual de folklore. La vida popular tradicional en España. Madrid: Istmo.

Lowenfeld, V. (1961). Desarrollo de la capacidad creadora. Buenos Aires: Kapelusz.

Marín Ibáñez, R. (1976). Valores, objetivos y actitudes en educación. Valladolid: Miñón.

Muñoz Justicia, J. (2004). Análisis cualitativo de datos textuales con ATLAS.ti 5. Universitat Autònoma de Barcelona. Juan Muñoz Justicia ${ }^{\odot}$. Disponible en: http://www.geocities.com/cibertlan/doc s/materiales/ManualAtlas5.pdf [consulta 2008, 22 de junio]

Nicholls, J.G. (1984). Achievement motivation: Conceptions of ability, subjective experience, task choice, and performance. Psychollogical Review, 91, 328-346.

Nicholls, J.G. (1989). The Competitive Ethos and Democratic Education. Cambridge, MA: Harvard University Press.

Oller, M.T. y Martí Mora, E. (1998). Panorámica de la música y la danza tradicional valenciana. Valencia: Universidad Politécnica de Valencia, D.L.

Omeñaca Cilla, R. y Ruiz Omeñaca, J.V. (1999). Juegos cooperativos y educación física. Barcelona: Paidotribo.

Oreja Rodríguez, J.R. (2005). Introducción a la medición objetiva en economía, administración y dirección de empresas: el modelo de Rasch. IUDESerie estudios, 47, 1-78. Disponible en: http://dialnet.unirioja.es/servlet/extaut?codigo $=269604$ [consulta 2008, 14 de mayo]

Orlick, T. (1997). Juegos y deportes cooperativos: desafíos divertidos sin competición. Madrid: Editorial Popular.
Preciado, D. (1969). Folklore español: música, danza y ballet. Madrid: Studium.

Pring, R. (2005). El arte y la finalidad moral de la educación, en VV.AA. Cultivar los sentimientos. Propuestas desde la Filosofía de la Educación, Madrid: Dykinson, 149-164.

Pujol Subirà, M. A. y Serra Vilamitjana, J. (1998). La dansa catalana en l'ensenyament primari, Eines de cultura popular $\mathrm{N}^{\mathrm{o}}$ 1, Tarragona: El Mèdol, Generalitat de Catalunya.

Read, H. (1986). Educación por el arte. Barcelona: Paidós.

Rodríguez Lloréns, R. (2005). La Competitividad en las clases del Grado Elemental de Danza. [Trabajo del periodo de investigación de Doctorado] (Inédito)

Rodríguez Lloréns, R. (2009). La educación en valores a través de la danza en las enseñanzas regladas y en el folklore. Propuesta educativa para el ámbito de los estudios oficiales de danza. [Tesis Doctoral] Disponible en:

http://e-spacio.uned.es/fez/view.php? pid=tesisuned:Educacion-Rrodriguez [consulta 2010, 5 de febrero]

Recommendation: $\mathrm{N}^{\circ} \mathrm{R}$ (98) 5 of the Committee of Ministers to Member States concerning Heritage Education.

Salazar, A. (2003). La danza y el ballet. Madrid: Fondo de Cultura Económica de España.

Sandín Esteban, M.P. (2003). Investigación cualitativa en educación: fundamentos y tradiciones. Madrid: McGrawHill Interamericana.

Seminar: Cultural heritage and its educational implications: a factor for tolerance, good citizenship and social integration. Council of Europe, Brussels (Belgium), 28-30 August 1995. 
Steeh, J. (1982). History of Ballet and Modern dance. Londres: Magna Books.

Vygotskii, L.S. (1982). La imaginación y el arte de la infancia: ensayo psicológico. Torrejón de Ardoz: Akal.

Velázquez Callado, C. (2004). Las actividades físicas cooperativas. Una pro- puesta para la formación en valores a través de la educación física en las escuelas de educación básica. Méjico: Secretaría de Educación Pública.

Wright, B.D. y Stone M.H. (1998). Diseño de mejores pruebas. Utilizando la técnica de Rasch. Méjico: Ceneval. 


\section{PALABRAS CLAVE}

Educación artística, Educación en valores, Danza, Danza popular, Teoría de respuesta al ítem, Observación Participante, Aprendizaje cooperativo, Grupos experimentales.

\section{KEY WORDS}

Art Education, Values Education, Dance, Folk Dance, Item Response Theory, Participant Observation, Cooperative learning, Experimental Groups.

\section{PERFIL ACADÉMICO DE LA AUTORA}

Doctora en Pedagogía y Licenciada en Ciencias Políticas y Sociología (Sección Sociología) por la UNED. Licenciada en Pedagogía de la Danza por el Conservatorio de Danza de Valencia. Profesora del Departamento de Pedagogía de la Danza del Conservatorio Superior de Danza de Valencia, del Instituto Superior de Enseñanzas Artísticas de la Comunitat Valenciana, ISEACV. Principales líneas de interés en la investigación: educación en valores a través de la danza y nuevas didácticas de la danza

Dirección de la autora: Dpto. Pedagogía de la Danza

Conservatorio Superior de Danza de Valencia Camino de Vera s/n. 46022-Valencia e-mail: r_rodriguezllorens@hotmail.com

Fecha de Recepción del Artículo: 05. Noviembre. 2009

Fecha de revisión del artículo: 18. Enero. 2010

Fecha de Aceptación del Artículo: 09. Marzo. 2010 
